\title{
HÁBITOS SALUDABLES EN MUJERES ADULTAS Y MADRES
}

\author{
Dunia Garrido \\ Departamento de Psicología, Área de Personalidad, Evaluación y Tratamiento \\ Psicológico. Universidad Castilla La Mancha. España \\ Dunia.garrido@uclm.es \\ Yolanda Sevilla Vera \\ Departamento de Psicología, Área de Psicología Evolutiva y del Desarrollo \\ Universidad de Castilla La Mancha, España \\ yolanda.sevilla@uclm.es \\ Natalia Solano Pinto \\ Departamento de Psicología, Área de Psicología Evolutiva y del Desarrollo \\ Universidad de Castilla La Mancha, España \\ natalia.solano@uclm.es
}

Recepción Artículo: 3 febrero 2020
Admisión Evaluación: 4 marzo 2020
Informe Evaluador 1: 10 m 2020
Informe Evaluador 2: 15 marzo 2020
Aprobación Publicación: 20 abril 2020

\section{RESUMEN}

Introducción. Según la Organización Mundial de la Salud, un índice de masa corporal (IMC) elevado es un importante factor de riesgo de enfermedades cardiovasculares, diabetes y algunos tipos de cáncer. Algunos de Ios factores protectores que se han asociado con un peso adecuado son el consumo de verduras, frutas y el bajo consumo de comida rápida. Además, algunos de estos factores (como el consumo de verdura) parece que presentan una relación positiva entre padres e hijos. Por ello, se hace necesario evaluar los hábitos saludables en madres de niños de edad escolar.

Objetivos. Caracterizar los hábitos saludables en cuidado de salud en madres y su posible relación con el peso.

Metodología. Estudio transversal en el que se realizó un análisis exploratorio sobre hábitos de vida saludables y su relación con el IMC a partir de un muestreo no probabilístico sobre una muestra de 100 madres con una media de edad de 43.07 (5.09). Se utilizó el cuestionario Autoeficacia en el cuidado de la salud, versión abreviada.

Resultados. Las correlaciones de Pearson muestran que no existe una correlación significativa entre los hábitos no saludables de cuidado de salud y presentar normopeso o sobrepeso. Sin embargo, sí existe una correlación significativa entre los hábitos saludables de cuidado de salud y presentar normopeso ( $r=.290, p<.01) 0$ sobrepeso $(r=-.249, p<.05)$ en las madres evaluadas.

Conclusiones. Nuestros datos sugieren que los hábitos de vida saludable pueden considerarse como un factor protector contra la obesidad en madres.

Palabras clave: hábitos saludables; factores de riesgo; sobrepeso; salud 


\section{HÁBITOS SALUDABLES EN MUJERES ADULTAS Y MADRES}

\section{ABSTRACT}

Introduction. According to World Health Organization, raised body mass index (BMI) is a major risk factor for cardiovascular diseases, diabetes, and some cancers. Some protective factors have been associated with healthy weight, such as consumption of fruit, vegetables, and a reduced consumption of processed food or junk food. Moreover, some of these factors (such as consumption of vegetables) might have a positive relationship between parents and children. Thus, it is necessary to evaluate healthy habits on mothers of school-aged children.

Objective. To define healthy habits on mothers, and its potential relationship with weight.

Methods. An exploratory analysis about healthy habits and its relationship with BMI was carried out in a cross-sectional study. Our sample consisted on 100 mothers with an average age of 43.07 (5.09). We used a brief version of the Self-efficacy in health care scale.

Results. Pearson's correlations showed no relationships between unhealthy habits of health care and normal or overweight. However, our results showed a significant relationship between healthy habits on health care and normal weight $(r=.290, p<.01)$ and overweight $(r=-.249, p<.05)$.

Conclusions. Our results suggest that healthy habits might be considered as a protective factor against overweight in mothers of school-aged children.

Keywords: healthy habits; risk factors; overweight; health.

Según la Organización Mundial de la Salud (OMS), un índice de masa corporal (IMC) elevado es un importante factor de riesgo de enfermedades cardiovasculares, diabetes y algunos tipos de cáncer (OMS, 2020). En Europa y en España, el $54 \%$ de las mujeres adultas presentan sobrepeso (IMC $\geq 25$ ) según el último informe publicado de la OMS (2016). En las últimas décadas, se está prestando especial atención en la promoción de estilos de vida saludables, dado que la efectividad de los tratamientos para el control de la obesidad no supera el 20\% (Campos et al., 2008). Por tanto, debido a la alta prevalencia de sobrepeso en nuestra sociedad y las graves consecuencias que implica presentar un IMC elevado para la salud, se hace necesario el analizar y ofrecer factores que ayuden a prevenir un elevado IMC.

A lo largo de la literatura se han analizado diversos factores de riesgo que contribuyen y facilitan la aparición de sobrepeso u obesidad. Algunos de estos factores de riesgo son ser hombre, el sedentarismo, y un alto consumo de bebidas azucaradas, alcohol, alimentos procesados de alta densidad energética, grasas saturadas, sal y azúcar (Andrade, 2016; Crovetto et al., 2018). Afortunadamente, también se han propuesto diversos factores que contribuyen y facilitan un buen estado de salud general de las personas (incluyendo un saludable IMC) y su bienestar. Por ejemplo, algunos de los factores que se han asociado con un peso adecuado y un bajo riesgo de presentar sobrepeso u obesidad son el consumo de alimentos de origen vegetal (como verduras, cereales integrales y frutas) y el bajo consumo de comida rápida o aperitivos fritos y dulces (Crovetto et al., 2018; Durán-Agüero et al., 2016).

Adicionalmente, otro de estos factores protectores que se ha estudiado a lo largo de la literatura son las habilidades de autoeficacia en el cuidado de la salud (Klein-Hessling et al., 2005). Se considera que la creencia sobre la eficacia en el cuidado de la salud es un potente predictor de dichas conductas de autocuidado (Thrasher et al., 2016), que promueven un estilo de vida activo y saludable. De esta forma, algunas investigaciones han descrito relaciones positivas entre la autoeficacia y una reducción del peso en adultos con sobrepeso, así como mantener el peso en personas adultas con normopeso (Amnesi y Tennant, 2014; Teixeira et al., 2010). Además, también se ha relacionado la autoeficacia con el consumo de alimentos bajos en calorías, frutas y verduras (Povey et al., 2000).

Siguiendo esta línea argumental, además de las consecuencias que a nivel personal conduce el presentar un elevado IMC, parece que en el caso de las madres también puede ejercer un impacto a nivel familiar (SolanoPinto et al., 2017). De hecho, aún se considera que la transmisión cultural de la forma de alimentación se da principalmente a través de la madre, por lo que las madres tendrían un rol fundamental en la educación y transmisión de pautas alimentarias desde edades muy tempranas (Kroke et al., 2006; Osorio et al., 2002). Así, estas investigaciones parecen indicar que la presencia de hábitos no saludables en la población adulta, y en concreto en las 
madres, podría tener importantes consecuencias en la salud y el bienestar de sus hijos. La importancia de este hecho radica en que los factores de riesgo que contribuyen al incremento de la morbilidad y mortalidad ya aparecen en edades tempranas, por lo que el tiempo de exposición a estos factores de riesgo en poblaciones jóvenes tendrían previsiblemente consecuencias a nivel epidemiológico.

Algunos estudios clásicos han mostrado que presentar déficits en las conductas relacionadas con la alimentación de las madres se relacionan con conductas posteriores que favorecen el sobrepeso y la obesidad en sus hijos (Campos et al., 1998). Sin embargo, también se han evaluado factores protectores que se incluyen dentro de los factores de autoeficacia en el cuidado de la salud. De forma específica, parece que el consumo de verdura presenta una relación positiva entre padres e hijos (Solano-Pinto et al., 2017). Esta relación parece explicarse bajo el efecto del aprendizaje social que implica imitar la conducta de los padres cuando se sientan a la mesa a comer (Solano-Pinto et al., 2017).

Sin embargo, desde nuestro conocimiento, no se han analizado las conductas relativas a la alimentación, como el autocuidado de la salud en madres. Por ello, se hace necesario evaluar los hábitos saludables en madres de niños de edad escolar. El objetivo del presente estudio es caracterizar los hábitos saludables en cuidado de salud en madres y su posible relación con el peso.

\section{MÉTODO}

\section{Participantes}

En este estudio han participado un total de 100 madres (media de 43.07 años de edad, rango de 24 a 53 años de edad) de alumnos de educación primaria de un centro educativo de la Toledo (España). La edad media de los/as hijos/as era de 9.90 años (rango de edad de 8 a 11 años). En relación con las características socioeducativas de la muestra, la mayor parte de las madres $(n=80,80 \%)$ contaban con un nivel educativo superior a estudios de segundo grado o estudios universitarios. De la muestra total, 85 madres (85\%) realizaban algún trabajo de tipo remunerado fuera del hogar (a tiempo completo y/o parcial).

El criterio de inclusión de las participantes fue que tuvieran un/a hijo/a escolarizado/a en un centro educativo de la ciudad de Toledo (España) y que aceptasen de forma voluntaria y anónima participar en la investigación. Las principales características sociodemográficas de la muestra de participantes se recogen en la Tabla 1.

Tabla 1. Variables socio-demográficas de las participantes del estudio

\begin{tabular}{lllll}
\hline & \multicolumn{4}{l}{ Madres de niños de primaria (N =100) } \\
\cline { 2 - 5 } & $\mathrm{N}(\%)$ & Media & DT & Rango \\
\hline Datos escolares & - & 9.90 & .91 & $8-11$ \\
Edad & $50(50 \%)$ & - & - & - \\
Género (masculino) & & & & \\
Datos madres & - & 43.07 & 5.09 & $24-53$ \\
Edad & & & & \\
Nivel educativo & $1(1 \%)$ & - & - & - \\
$\quad$ Sin estudios & $17(17 \%)$ & - & - & - \\
$\quad$ Educación primaria & $5(5 \%)$ & - & - & - \\
$\quad$ Educación secundaria & $98(98 \%)$ & - & - & - \\
$\quad$ Educación superior & & & & \\
Situación laboral & $4(5 \%)$ & - & - & - \\
$\quad$ En paro & $11(11 \%)$ & - & - & - \\
$\quad$ Ama de casa & $85(85 \%)$ & - & - & - \\
$\quad$ Trabaja & & &
\end{tabular}




\section{HÁBITOS SALUDABLES EN MUJERES ADULTAS Y MADRES}

\section{Instrumentos}

Índice de masa corporal. La evaluación antropométrica del peso y la altura se realizó a través de un cuestionario donde las participantes informaron acerca de estas dos medidas. Tras la recogida de estos datos, se calculó para cada una el índice de masa corporal (IMC; $\left.\mathrm{kg} / \mathrm{m}^{2}\right)$. Se clasificaron a las participantes en función de si presentaban normopeso $(\mathrm{IMC}<25)$ o sobrepeso $(\mathrm{IMC} \geq 25)$.

Autoeficacia en el cuidado de la salud. Para evaluar la autoeficacia en el cuidado de la salud se ha utilizado la versión abreviada de la Escala de autoeficacia en el cuidado de la alimentación y salud física (EACASF; Blanco et al., 2016). Es una escala de tipo Likert de 10 puntos en la que se responde si se siente capaz de realizar cada una de las conductas relacionadas con el cuidado de la salud (donde 0 significa nada capaz y 10 significa totalmente capaz). En concreto para este estudio, hemos analizado la versión breve de la escala, en la que se evalúan tanto hábitos saludables (formada por 3 ítems; p.ej., realización de actividad física e ingesta alimentaria equilibrada) como no saludables (formada por 6 ítems; p.ej., consumo de comida rápida, tabaco, alcohol y estrés). Ambos factores han mostrado valores aceptables en ciencias sociales respecto a la consistencia interna en nuestra muestra, con un Alfa de Cronbach de .65 y de .55 respectivamente.

\section{Procedimiento}

En el estudio se tuvo en cuenta la declaración de Helsinki. Además, se informó de los objetivos de la investigación y se solicitó el consentimiento informado a los centros educativos. Una vez obtenido dicho consentimiento por parte de los centros educativos, el propio centro informaba y solicitaba la participación garantizando el anonimato y la voluntariedad en dicha participación. Se les facilitaba los cuestionarios en un sobre cerrado a través del tutor de curso y devolvían el sobre cerrado a la secretaría del centro. La evaluación de cada participante se llevó a cabo a través de la entrega de los cuestionarios a las familias.

\section{Análisis de los datos}

Se han realizado análisis descriptivos para caracterizar la muestra en las diversas variables objeto de estudio de este trabajo (IMC, hábitos saludables y no saludables relacionados con el autocuidado de la salud). Posteriormente se realizaron correlaciones bivariadas de Pearson para determinar la posible relación entre el IMC y los hábitos de autocuidado de la salud. Todos Ios análisis se realizaron utilizando el paquete estadístico SPSS versión 25.0.

\section{RESULTADOS}

La media del IMC de las madres evaluadas fue de 22.79. La mayor parte de las madres (73\%) mostró tener un IMC dentro de los rangos considerados como peso saludable o normopeso $(\mathrm{IMC}<25)$. En relación con los hábitos no saludables de autocuidado de salud, un 92\% obtuvo puntuaciones por debajo de 30, mostrando que la mayoría de las madres evaluadas presentaban un patrón bajo de respuestas en dichos hábitos. Por el contrario, en relación con los hábitos saludables de autocuidado de salud, un 74\% de las madres evaluadas obtuvo puntuaciones por encima de 15, mostrando un patrón alto de respuestas en los hábitos protectores de autocuidado de salud. La representación de las puntuaciones obtenidas en los hábitos no saludables y saludables de autocuidado en función del IMC de las madres aparecen en las Figuras 1 y 2. 
PSICOLOGÍA Y CRECIMIENTO HUMANO

Tabla 2. Análisis descriptivo de las variables principales objeto de estudio

\begin{tabular}{lcccc}
\hline & \multicolumn{4}{c}{ Madres de niños de primaria $(\mathrm{N}=100)$} \\
\cline { 2 - 5 } & Media & DT & Min-max & Rango \\
\hline IMC, $\mathrm{kg} / \mathrm{m}^{2}$ & 22.79 & 3.58 & - & $17.65-37.34$ \\
Normopeso $(\mathrm{n}=73)$ & 21.73 & 1.76 & - & $18.78-24.86$ \\
$\quad$ Sobrepeso $(\mathrm{n}=17)$ & 28.51 & 3.79 & - & $25.10-37.34$ \\
Autoeficacia cuidado salud & & & & \\
$\quad$ Hábitos no saludables & 17.13 & 8.62 & $6-60$ & $3-46$ \\
Hábitos saludables & 20.06 & 6.76 & $3-30$ & $4-30$ \\
\hline
\end{tabular}

Figura 1. Hábitos no saludables de autocuidado de la salud en madres con normopeso y madres con sobrepeso.

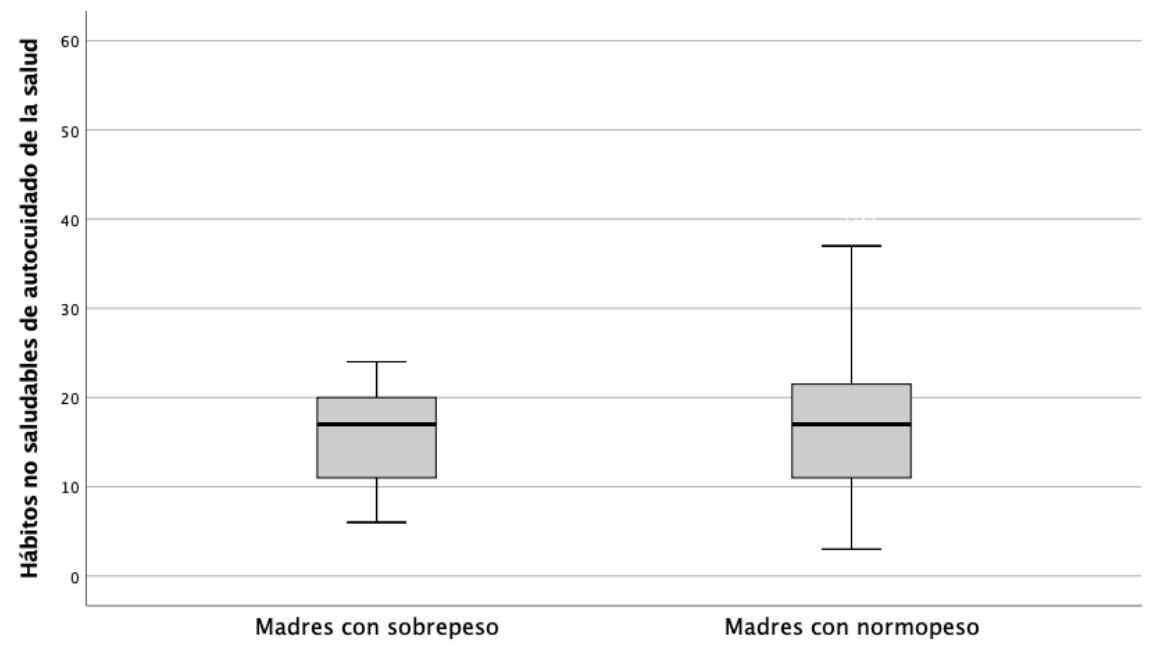




\section{HÁBITOS SALUDABLES EN MUJERES ADULTAS Y MADRES}

Figura 2. Hábitos saludables de autocuidado de la salud en madres con normopeso y madres con sobrepeso.

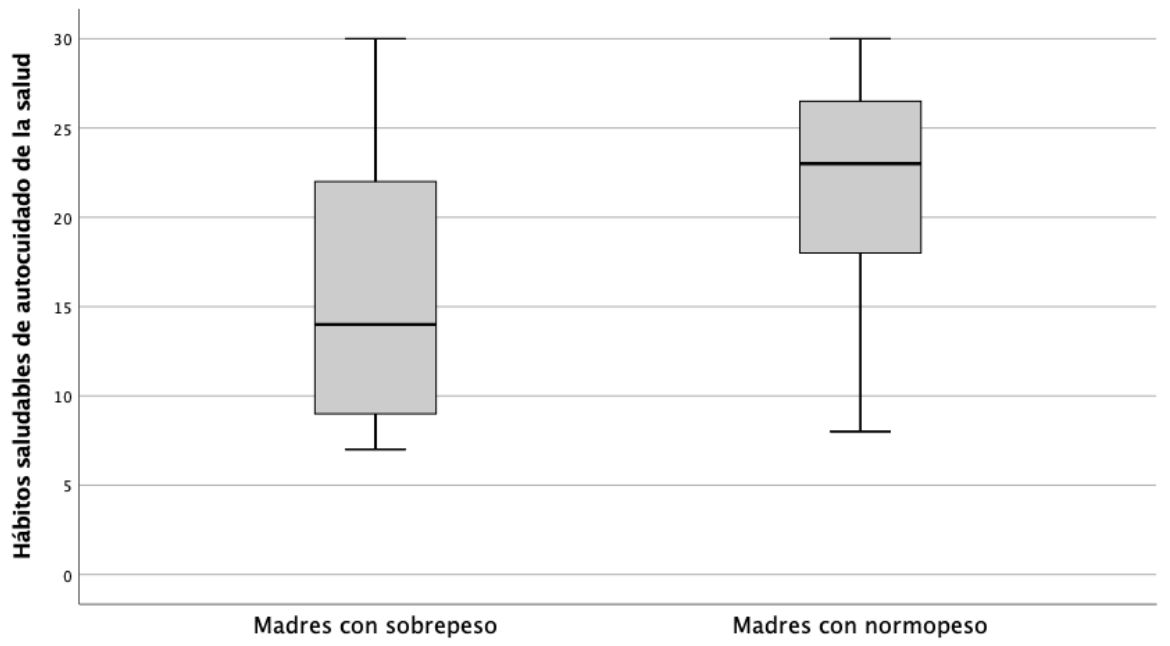

Las correlaciones de Pearson (ver Tabla 3) muestran que no existe una correlación significativa entre los hábitos no saludables de cuidado de salud y presentar normopeso o sobrepeso (todas con p >.05). Sin embargo, sí existe una correlación significativa entre los hábitos saludables de cuidado de salud y presentar normopeso 0 sobrepeso en las madres evaluadas (ambas con $p<.05$ ) (ver Tabla 3 y Figura 1).

Tabla 3. Correlaciones de Pearson entre el IMC y los hábitos de autocuidado de salud.

\begin{tabular}{lrrrrr}
\hline & 1 & 2 & 3 & 4 & 5 \\
\hline 1. IMC & - & & & & \\
2. Normopeso & $\mathbf{- . 5 5 4}$ & - & & & \\
3. Sobrepeso & $\mathbf{. 7 5 5}$ & $\mathbf{- . 8 7 6}$ & - & & \\
4. Hábitos no saludables & -.039 & .020 & -.045 & - & \\
5. Hábitos saludables & -.053 & $\mathbf{. 2 9 0}$ & $\mathbf{- . 2 4 9}$ & -.132 & -
\end{tabular}

Nota: IMC, índice de masa corporal; normopeso, IMC $<25$; sobrepeso, IMC $\geq 25$; las correlaciones significativas $(\mathrm{p}<.05)$ aparecen en negrita.

\section{DISCUSIÓN}

El objetivo principal de este estudio era el de caracterizar los hábitos saludables de cuidado de salud en madres de niños escolares y su potencial relación con el peso de la madre. Del análisis de nuestros datos se desprende que los hábitos saludables relacionados con la autoeficacia percibida en el cuidado de la salud (p.ej., la realización de actividad física y la ingesta alimentaria equilibrada) pueden considerarse como un factor protector contra el sobrepeso en madres. Sin embargo, en relación a los hábitos no saludables (p.ej., el consumo de comida rápida, tabaco, alcohol, o experimentar estrés), nuestros resultados parecen indicar que no existe una relación con el normopeso o con el sobrepeso de las madres evaluadas. 
En relación con los resultados obtenidos en los hábitos de vida saludable, nuestros datos concuerdan con los obtenidos por otros autores. En este sentido, nuestro estudio parece apoyar los resultados hallados por Amnesi y Tennant (2014) y Teixeira et al. (2010), donde describen relaciones positivas entre la autoeficacia y un mantenimiento del peso en adultos con normopeso y una relación negativa con el peso en adultos con sobrepeso. Además, nuestros resultados se muestran en la línea de otros trabajos donde se afirma que consumir alimentos de origen vegetal y el bajo consumo de comida rápida (todos ellos considerados dentro de una alimentación equilibrada) se asocian con un adecuado peso (Crovetto et al., 2018; Durán-Agüero et al., 2016).

Sin embargo, nuestros resultados parecen no estar en la línea de otros estudios que sugieren que diversos factores de riesgo están relacionados con el sobrepeso o la obesidad. Por ejemplo, Andrade (2016) y Crovetto et al. (2018) describieron algunos de esos factores de riesgo, como el sedentarismo y un alto consumo de bebidas azucaradas, alcohol, alimentos procesados, grasas saturadas, sal y azúcar. Una potencial explicación a este hecho podría estar en la dificultad para reconocer hábitos no saludables de autocuidado de la salud, dado que en la actualidad se encuentran muy castigados por la sociedad.

Una potencial explicación en la que descansarían nuestros resultados viene motivada por el hecho de que la creencia sobre la propia eficacia en el cuidado de la salud predice las conductas de autocuidado en la realidad (Thrasher et al., 2016). De nuestros resultados se desprende la importancia de establecer y promover hábitos de vida saludables, tal y como afirman otros autores (ver Campos et al., 2008). Por ello, consideramos que se deberían promover desde las instituciones públicas estilos de vida activos y saludables.

Sin embargo, estos resultados deben entenderse a la luz de ciertas limitaciones. En primer lugar, en nuestro estudio no hemos analizado conductas que favorecen el sobrepeso y la obesidad en los hijos, por lo que no se han podido llevar a cabo análisis para establecer relaciones entre la autoeficacia en el cuidado de la salud de las madres y los hijos. Por tanto, se deben realizar investigaciones futuras en las que se permita analizar dicha relación entre madres e hijos. En segundo lugar, dadas las características de nuestra muestra, existen limitaciones para extrapolar y generalizar los resultados obtenidos. Sería de un gran interés realizar otros estudios con muestras de mayor tamaño y con diversos niveles educativos y laborales, debido a que nuestra muestra estaba compuesta principalmente por madres con un nivel educativo superior y que se encuentran realizando trabajos remunerados. Por último, también sería importante evaluar en estudios futuros si estos resultados también se obtienen cuando se evalúa la autoeficacia en el cuidado de la salud en padres de niños de edad escolar y la potencial relación con los hábitos de vida saludable de sus hijos.

\section{CONCLUSIONES}

En suma, como resultado de este trabajo, nuestros datos sugieren que los hábitos de vida saludable pueden considerarse como un factor protector contra la obesidad en madres. Por lo que atender y promover las habilidades saludables de autoeficacia en el cuidado de la salud en madres de niños escolares se materializa como un elemento protector contra un IMC elevado.

\section{REFERENCIAS BIBLIOGRÁFICAS}

Andrade, M. A. (2017). Autocuidado y patrones de consumo, asociados al sobrepeso, obesidad en adultos, CLAS San Juan Bautista, Ayacucho 2016. Revista Investigación, 25(2), 133-141.

Annesi, J. J., y Tennant, G. A. (2014). Generalization of theory-based predictions for improved nutrition to adults with morbid obesity: Implications of initiating exercise. International Journal of Clinical and Health Psychology, 14(1), 1-8. Doi:

Blanco, L. H.., Díaz-Leal, A. C., Contreras, M. O., Mondaca, F., y Solano-Pinto, N. (2019). La Autoeficacia en el Cuidado de la Salud en la Predicción de la Satisfacción con la Vida. Revista iberoamericana de diagnóstico y evaluación psicológica, 3(52), 53-66. doi: 10.21865/RIDEP52.3.05

Campos, C., Jaimovich, S., y Campos, M. S. (1998). Actitudes de crianza en relación a tres problemas de salud: Comparación de dos grupos de embarazadas atendidas bajo diferente modalidad. Ciencia y Enfermería, 4(1), 29-35. 


\section{HÁBITOS SALUDABLES EN MUJERES ADULTAS Y MADRES}

Campos, C., Jaimovich, S., Moore, J., Pawloski, L., Gaffney, K., y González, M. (2008). Operaciones de Autocuidado asociadas a la nutrición en madres de escolares chilenos. Horizon Enferm, 19(2), 45-54.

Crovetto, M., Valladares, M., Espinoza, V., Mena, F., Oñate, G., Fernandez, M., y Durán-Agüero, S. (2018). Effect of healthy and unhealthy habits on obesity: a multicentric study. Nutrition, 54, 7-11. Doi: 10.1016/j.nut.2018.02.003

Durán-Agüero, S., Fernández-Godoy, E., Fehrmann-Rosas, P., Delgado-Sánchez, C., Quintana-Muñoz, C., YungeHidalgo, W., Hidalgo-Fernández, y Fuentes-Fuentes, J. (2016). Menos horas de sueño asociado con sobrepeso y obesidad en estudiantes de nutrición de una universidad chilena. Revista Peruana de Medicina Experimental y Salud Pública, 33, 264-268. doi: 10.17843/rpmesp.2016.332.210

Klein-Hessling, J., Lohaus, A., y Ball, J. (2005). Psychological predictors of health-related behaviour in children. Psychology, Health \& Medicine, 10(1), 31-43. doi: 10.1080/1354850051233131534

Kroke, A., Strathmann, S., y Günther, A. L. (2006). Maternal perceptions of her child's body weight in infancy and early childhood and their relation to body weight status at age 7. European Journal of Pediatrics, 165(12), 875-883. Doi: 10.1007/s00431-006-0191-3

Organización mundial de la salud (2016). Prevalence of overweight among adults, $B M l \geq 25$, age-standardized. Estimates by WHO Region. Disponible en https://apps.who.int/gho/data/view.main.GLOBAL2461A?lang=en. Consultado el 2 Abril, 2020.

Organización mundial de la salud (2020). Obesidad y sobrepeso. Disponible en https://www.who.int/es/newsroom/fact-sheets/detail/obesity-and-overweight. Consultado el 2 Abril, 2020.

Osorio, E., Weisstaub, N., y Castillo, D. (2002). Desarrollo de la conducta alimentaria en la infancia y sus alteraciones. Revista Chilena de Nutrición, 29(3), 280-285. Doi: 10.4067/S0717-75182002000300002

Povey, R., Conner, M., Sparks, P., James, R., y Shepherd, R. (2000). Application of the Theory of Planned Behaviour to two dietary behaviours: Roles of perceived control and self efficacy. British Journal of Health Psychology, 5(2), 121-139. Doi: 10.1348/135910700168810

Solano-Pinto, N., Solbes-Canales, I., Fernández-Cézar, R., Calderón-López, S., y Pozo-Bardera, C. (2017). Healthy habits in preschoolers and their families. An invitation to reflection. Demetra: Food, Nutrition \& Health, 12(4). doi: 10.12957/demetra.2017.28657

Teixeira, P. J., Silva, M. N., Coutinho, S. R., Palmeira, A. L., Mata, J., Vieira, P. N., Carraca, E. V., Santos, T. C., y Sardinha, L. B. (2010). Mediators of weight loss and weight loss maintenance in middle aged women. Obesity, 18(4), 725-735. Doi: 10.1038/oby.2009.281

Thrasher, J. F., Swayampakala, K., Borland, R., Nagelhout, G, Young, H., Hammond, D., Bansal-Travers, M., Thompson, M., y Hardin, J. (2016). Influence of self- efficacy, and reactance on responses to cigarette health warnings: a longitudinal study of adult smokers in Australia and Canada. Health Communication, 31(12), 1517-1526. Doi: 10.1080/10410236.2015.1089456 